\title{
Lesão do tendão do músculo supraespinal e sua relação com a lesão do tendão da cabeça longa do bíceps*
}

\section{Supraspinatus Muscle Tendon Lesion and Its Relationship with Long Head of the Biceps Lesion}

\author{
Cassiano Diniz Carvalho ${ }^{10}$ Carina Cohen ${ }^{1}$ Paulo Santoro Belangero ${ }^{1}$ Alberto de Castro Pochini ${ }^{1}$ \\ Carlos Vicente Andreoli ${ }^{1}$ Benno Ejnisman ${ }^{1}$ \\ ${ }^{1}$ Departamento de Ortopedia e Traumatologia, Centro de \\ Endereço para correspondência Cassiano Diniz Carvalho, Rua \\ Traumatologia do Esporte (CETE), Escola Paulista de Medicina, \\ Universidade Federal de São Paulo (Unifesp), São Paulo, SP, Brasil \\ Teixeira Santana 185, Fundinho, Uberlândia, 38400-196, Brasil \\ (e-mail: cassianodiniz@labsforfit.com.br).
}

Rev Bras Ortop 2020;55(3):329-338.

\begin{abstract}
Resumo
Objetivo Identificar a correlação clínica, radiológica, e artroscópica das lesões do tendão da cabeça longa do bíceps e sua influência na dor do paciente quando associada às lesões do manguito rotador.

Métodos Entre abril e dezembro de 2013, foram avaliados 50 pacientes, sendo 38 (76\%) do sexo feminino e 12 (24\%) do sexo masculino, com idade média de 65,1 anos. Os pacientes foram operados pelo Grupo de Ombro e Cotovelo da Disciplina de Medicina Esportiva do Departamento de Ortopedia e Traumatologia da Universidade Federal de São Paulo. Os indivíduos foram submetidos a reparo da lesão do manguito rotador com evidência clínica, radiológica e/ou artroscópica de acometimento do tendão da cabeça longa do bíceps.

Resultados Observou-se associação entre dor à palpação do sulco intertubercular do úmero com lesão parcial de alto grau (ruptura parcial acometendo mais de $50 \%$ do tendão) na artroscopia $(p=0,003)$. Encontramos ainda uma associação entre a lesão de

Palavras-chave

- cabeça longa do bíceps

- tendinopatia

- manguito rotador

- tenotomia

- tenodese alto grau da cabeça longa do bíceps e a lesão do tendão do músculo supraespinal $(p<0,05)$, sendo que, para cada centímetro de lesão do tendão do músculo supraespinal, o paciente apresenta probabilidade 1,7 maior de ter uma lesão de alto grau da cabeça longa do bíceps.

Conclusão A dor na região anterior do ombro à palpação do sulco intertubercular do úmero pode estar relacionada às lesões de alto grau da cabeça longa do bíceps. A lesão do manguito rotador e o seu tamanho são fatores de risco para lesão de alto grau do tendão da cabeça longa do bíceps.
\end{abstract}

Trabalho feito no Centro de Traumatologia do Esporte (CETE), Departamento de Ortopedia e Traumatologia, Escola Paulista de Medicina, Universidade Federal de São Paulo (Unifesp), São Paulo, SP, Brasil.

recebido

21 de Julho de 2018

aceito

05 de Fevereiro de 2019
DOI https://doi.org/

10.1055/s-0039-3402472. ISSN 0102-3616.
Copyright $\odot 2020$ by Sociedade Brasileira License terms de Ortopedia e Traumatologia. Published by Thieme Revinter Publicações Ltda, Rio de Janeiro, Brazil 


\begin{abstract}

\section{Keywords}

- long head of the biceps

- tendinopathy

- rotator cuff

- tenotomy

- tenodesis

Objective To identify the clinical, radiological, and arthroscopic correlation of long head of the biceps tendon injuries and their influence on pain when associated with rotator cuff injuries.

Methods Between April and December 2013, 50 patients were evaluated, including 38 (76\%) women and $12(24 \%)$ men, with a mean age of 65.1 years old. The patients were operated by the Shoulder and Elbow Group, Discipline of Sports Medicine, Orthopedics and Traumatology Department, Universidade Federal de São Paulo. The subjects underwent repair of the rotator cuff lesion with clinical, radiological and/or arthroscopic evidence of involvement of the long head of the biceps tendon.

Results An association between pain at palpation of the intertubercular groove of the humerus and high-grade partial lesions (partial rupture of the tendon affecting more than $50 \%$ of its structure) was observed at the arthroscopy $(p=0.003)$. There was also an association between the high-grade lesion of the long head of the biceps and injury to the supraspinatus muscle tendon $(p<0.05)$. For each centimeter of the supraspinatus muscle tendon injury, the patient presented a 1.7 higher probability of having a high-grade lesion at the long head of the biceps.

Conclusion Pain at the anterior shoulder region during palpation of the intertubercular groove of the humerus may be related to high-grade lesions to the long head of the biceps. Rotator cuff injury and its size are risk factors for high-grade injuries to the long head of the biceps tendon.
\end{abstract}

\section{Introdução}

O tendão da cabeça longa do bíceps (CLB) braquial é uma estrutura que, ao longo da história, sempre foi objeto de grande controvérsia, sendo considerada desde uma importante fonte de dor no ombro até uma estrutura sem nenhuma importância. ${ }^{1}$ Kessell e Watson ${ }^{2}$ a descreveram como uma estrutura fácil de se culpar, mas difícil de se condenar.

Ainda não há um consenso sobre o verdadeiro papel do tendão da CLB na biomecânica do ombro. Apesar disso, a sua função e o seu importante papel na estabilização estática e dinâmica vêm sendo investigados por vários autores. ${ }^{3}$ Diversos autores observaram o importante papel estabilizador, ${ }^{4}$ bem como a centralização da cabeça umeral na glenoide secundária, conforme descritos por Pagnani et al. ${ }^{5}$

Em 1934, a tendinite do bíceps foi questionada por Codman, ${ }^{6}$ que chegou a duvidar do processo inflamatório no tendão, achando bem mais provável que a causa da dor se devesse às lesões do tendão do músculo supraespinal. 0 autor não conseguiu provar o envolvimento do bíceps em nenhum de seus casos. A partir dos anos 50, diversos autores consideravam a tendinite do bíceps uma importante causa da dor no ombro, e tratavam-na com tenodese. ${ }^{2,7}$ Em 1950, DePalma $^{7}$ descreveu alterações degenerativas do tendão e tratamento conservador e cirúrgico das lesões. Já em 2015, Godinho et $\mathrm{al}^{8}$ descreveram uma nova técnica cirúrgica para a tenodese da CLB.

O tendão da CLB tem $9 \mathrm{~cm}$ de comprimento, ${ }^{9}$ e é dividido em uma parte intra-articular e outra extra-articular, fibrocartilaginosa, que seria a parte do tendão na área de deslizamento no sulco intertubercular do úmero. Vale a pena ressaltar que essa divisão não tem $100 \%$ de acurácia. A cabeça umeral se move em relação ao tendão como em um trilho, e este, por sua vez, não se move em relação ao sulco bicipital. ${ }^{2,10}$ Portanto, a posição do braço é que irá ditar a relação entre as porções intra e extra-articulares. Por exemplo, em adução e extensão do braço, a maior parte do tendão encontra-se no interior da articulação. Em contrapartida, nos extremos da abdução, apenas uma pequena parte do tendão encontra-se no interior da articulação. ${ }^{1}$

Devido às características citadas anteriormente, a tendinopatia da CLB pode surgir em função da pressão e das forças de cisalhamento provocadas pelas repetidas fricções, trações e rotações glenoumerais. $O$ sulco intertubercular, por ser um ambiente constrito, geralmente permite que o processo inflamatório acometa essa região. ${ }^{11}$

Os testes diagnósticos para as lesões do tendão da CLB, quando aplicados individualmente, apresentam uma utilidade clínica limitada. $^{12}$

Atualmente, o exame complementar mais utilizado para o diagnóstico das lesões do tendão da CLB é a ressonância magnética. Os estudos apontam uma sensibilidade que varia de $52 \%$ a $69,8 \%$, e uma especificidade que varia de 86 a $98 \%$ para as lesões completas. ${ }^{13}$ Todavia, a avaliação durante o procedimento artroscópico é considerada o padrão-ouro para o diagnóstico das lesões intra-articulares do tendão da CLB. ${ }^{14}$

Neviaser et $a 1^{15}$ observaram, em seu estudo, uma íntima relação entre a tendinopatia da CLB e as lesões do manguito rotador por meio de artrografia e alterações macroscópicas no intra-operatório.

O objetivo do presente estudo foi avaliar a correlação clínica, radiológica, e artroscópica das lesões do tendão da 
CLB associadas às lesões do manguito rotador, e sua relação com a dor referida na região anterior do ombro.

\section{Materiais e Métodos}

Entre abril de 2013 e dezembro de 2013, foram avaliados e operados 56 pacientes pelo cirurgião integrante do Grupo de Ombro e Cotovelo da Disciplina de Medicina Esportiva do Departamento de Ortopedia e Traumatologia da Universidade Federal de São Paulo. Para a realização deste estudo observacional e transversal, foram excluídos 6 pacientes da amostra inicial de 56 pacientes. Dois apresentaram o exame de ressonância magnética incompleto, três não apresentaram lesão do tendão da CLB no intraoperatório, e um paciente não concordou em assinar o termo de consentimento livre e esclarecido (TCLE). Entre os 50 pacientes restantes, 38 (76\%) eram do sexo feminino, e 12 (24\%), do sexo masculino, com idade média de 65,1 anos.

Os critérios de inclusão foram pacientes com queixa de dor na região anterior do ombro, submetidos ao reparo da lesão do manguito rotador, e com evidência clínica, radiológica e/ou artroscópica de acometimento do tendão da CLB.

Os critérios de exclusão foram pacientes submetidos ao reparo da lesão de manguito rotador e sem indicação de tenotomia devido à ausência de sintomatologia provocada pelas lesões da CLB ou, ainda, ausência de lesão no momento da artroscopia, e pacientes sem lesão no manguito rotador associada à lesão da CLB.

Os pacientes foram questionados e examinados por especialistas, membros da Sociedade Brasileira de Cirurgia do Ombro e Cotovelo (SBCOC). As ressonâncias magnéticas foram realizadas no equipamento Achieva 1.5T (Philips, Amsterdã, Holanda) e avaliadas por dois cirurgiões experientes, também integrantes da SBCOC, de acordo com os mesmos critérios especificados na folha da coleta; foi utilizada a classificação de Patte e a quantificação em centímetros da lesão do manguito rotador para a definição do grau de retração tendínea.

Os pacientes foram postos em posição de cadeira de praia, sob anestesia geral e bloqueio do plexo braquial, e submetidos a artroscopia para reparo da lesão do manguito rotador e avaliação intra e extra-articulares do tendão da CLB por meio de análise macroscópica das lesões. Com a óptica no portal posterior e o gancho artroscópico no portal anterior, promovemos o deslocamento da CLB no sentido inferior, trazendo a porção extra-articular do tendão para o interior da articulação.

A ponta do gancho artroscópico serviu de instrumento de medida para graduarmos a espessura acometida na lesão da CLB. A aferição foi realizada a $1,5 \mathrm{~cm}$ da inserção labial do tendão da CLB.

As ressonâncias magnéticas foram realizadas utilizando a técnica de eco de rotação com ponderações em T1 e T2. As variáveis analisadas ao exame foram: sinais de lesão da CLB, presença e tamanho da lesão do manguito rotador (utilizando a classificação de Patte), e presença de lesões associadas, como lesão labral superior de anterior para posterior (superior labral tear from anterior to posterior, SLAP) tipo II.

No intraoperatório, foram avaliadas as lesões macroscópicas do tendão da CLB, tais como: hiperemia, fibrilação, acha- tamento, lesão parcial, luxação do tendão, lesão da polia, lesão SLAP tipo II e, ainda, a lesão dos tendões do manguito rotador, inclusive do subescapular, que tem relação direta com a luxação da CLB e suas lesões.

O presente estudo foi aprovado pelo Comitê de Ética em Pesquisa (CEP) do Hospital São Paulo, e todos os pacientes incluídos assinaram o TCLE.

As variáveis numéricas foram expressas por médias e desvios padrão (DPs), medianas e quartis (Qs), e valores mínimos e máximos; as categóricas, por frequências absolutas e relativas.

As associações entre a presença de dor e os testes do exame físico, os achados da ressonância magnética e os achados intraoperatórios foram avaliadas por modelos de regressão logística simples.

As análises foram realizadas usando o programa Statistical Package for the Social Sciences (SPSS, SPSS, Inc., Chicago, IL, EUA), versão 18, adotando-se o nível de significância de 5\%.

Para avaliar a concordância e a discordância entre as lesões do tendão da CLB observadas nos exames físicos, na ressonância magnética e na artroscopia, especificamente entre a lesão parcial de alto grau da CLB e a lesão SLAP, aplicamos o teste não paramétrico de McNemar. Da mesma forma, adotamos o nível de significância de $5 \%$.

\section{Resultados}

A amostra final de análise foi composta por 50 pacientes sintomáticos submetidos a reparo de lesão do manguito rotador com sintomas e/ou exame de ressonância magnética que indicavam o acometimento do tendão da CLB.

O tempo dos sintomas variou entre 2 e 240 meses, e metade dos pacientes apresentavam sintomas havia pelo menos 12 meses. Em 70\% deles, o ombro direito foi acometido, e em 72\%, o membro acometido foi 0 dominante.

No exame físico, metade dos pacientes tinham uma amplitude de flexão ativa de até $160^{\circ}\left(\mathrm{Q} 1=140^{\circ}\right.$ e $\left.\mathrm{Q} 3=180^{\circ}\right)$ e passiva de até $180^{\circ}\left(\mathrm{Q} 1=170^{\circ}\right.$ e $\left.\mathrm{Q} 3=180^{\circ}\right)$. Observou-se que $66 \%$ deles apresentavam dor à palpação do tendão da CLB no sulco intertubercular do úmero. Já nos testes especiais, $72 \%$ dos pacientes tiveram resultados positivos no teste de O'Brien, 78\%, no teste de Palm Up, e 40\%, no teste de Yergason.

Na avaliação pela ressonância magnética, 92\% tinham tendinopatia no bíceps, e 1 paciente apresentou ruptura total (2,0\%). Quanto à lesão do tendão do músculo supraespinal, 4\% apresentaram lesão parcial bursal; 4\% apresentaram lesão parcial intra-articular; e os demais tinham lesão total do tendão, com retração tendínea variando entre $0,9 \mathrm{~cm}$ e $5 \mathrm{~cm}$, e mediana de $2,9 \mathrm{~cm}$.

Ainda na avaliação dos exames de imagem, $42 \%$ dos pacientes apresentaram lesão do tendão do músculo infraespinhal; $32 \%$, lesão do tendão do músculo subescapular; $14 \%$, luxação do tendão da CLB; e 4\%, lesão SLAP tipo II.

Durante a artroscopia, observou-se que 60\% dos pacientes tinham uma lesão do tendão da CLB com acometimento superior a $50 \%$ da espessura do tendão. Entre as alterações encontradas, $88 \%$ dos pacientes tinham hiperemia (- Figura 1); 


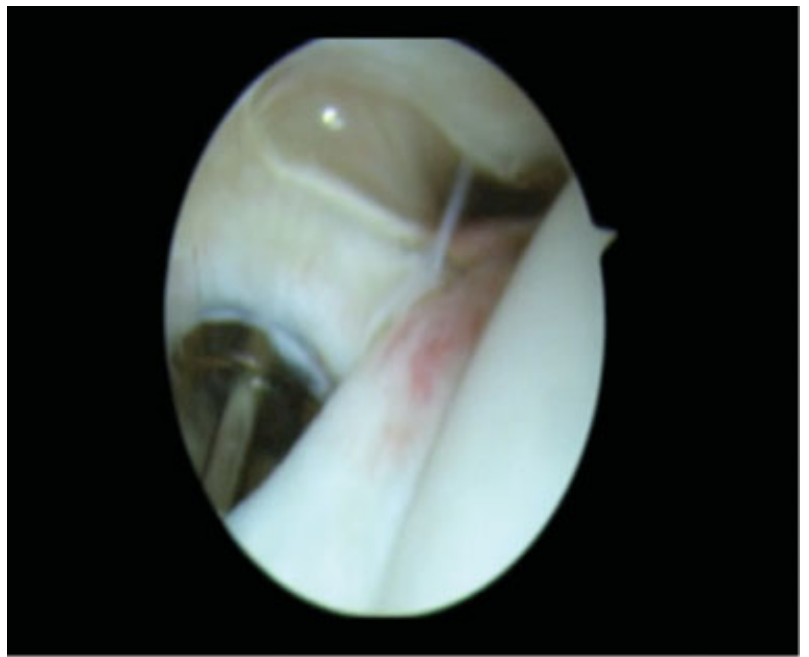

Fig. 1 Hiperemia do tendão da cabeça longa do bíceps (CLB).

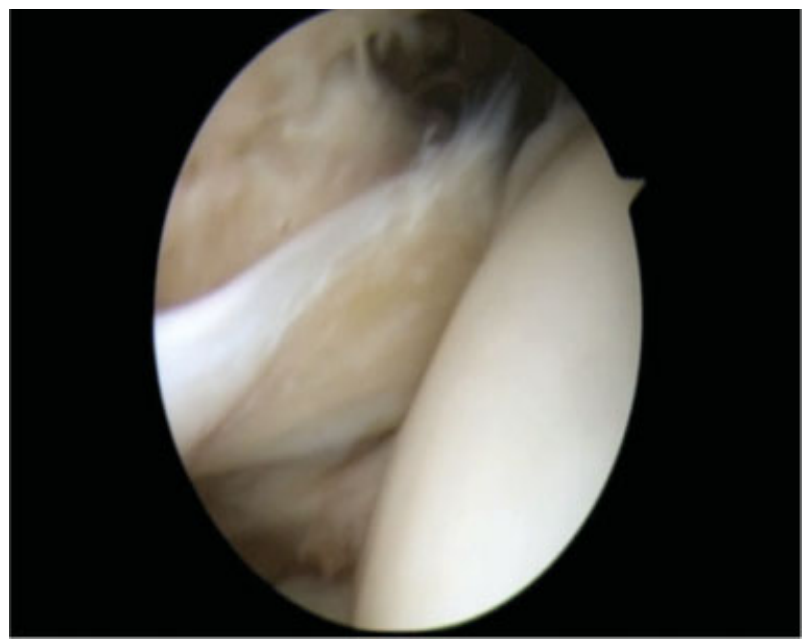

Fig. 2 Achatamento do tendão da cabeça longa do bíceps (CLB).

54\%, achatamento (-Figura 2); 86\%, fibrilação; 60\%, ruptura parcial; e 20\%, luxação do bíceps.

Foram observadas outras lesões na artroscopia relacionadas ao tendão da CLB: $24 \%$ dos pacientes apresentaram lesão do tendão do músculo subescapular; 22\%, lesão da polia; e $6 \%$, lesão SLAP tipo II.

Na - Tabela 1 foram apresentados a análise descritiva das variáveis numéricas da avaliação clínica, o exame físico, e os achados na avaliação radiológica na amostra de 50 pacientes.

$\mathrm{Na}$ - Tabela 2 foram apresentadas a distribuição entre os sexos, a dominância do membro acometido, e as variáveis categóricas do exame físico na amostra de 50 pacientes.

$\mathrm{Na}$ - Tabela 3 foram apresentados os achados sobre a avaliação radiológica (ressonância magnética), com relação à presença de lesão do tendão da CLB, à luxação do mesmo tendão em relação à goteira bicipital, e às lesões associadas do manguito rotador e lesão SLAP.

$\mathrm{Na}$ - Tabela 4 apresentamos as características morfológicas do tendão da CLB, a luxação do mesmo tendão em relação à goteira bicipital, a lesão associada do tendão do músculo subescapular e a lesão SLAP, observadas durante o procedimento artroscópico.

Observamos evidências de associação entre a presença de dor à palpação do sulco intertubercular do úmero e a lesão parcial de alto grau da CLB (ruptura parcial acometendo mais de $50 \%$ do tendão da CLB - - Figuras 3 e 4) na artroscopia $(p=0,003)$. Nos pacientes com dor à palpação do sulco intertubercular do úmero, foi observada uma probabilidade de $83 \%$ de eles apresentarem uma lesão significativa da CLB, ou seja, um acometimento maior do que $50 \%$ da espessura do tendão. Nessa perspectiva, os pacientes com resultado positivo nesse teste têm um risco 1,7 vezes maior de desenvolverem uma lesão significativa, diferente do que ocorre com os pacientes que têm resultado negativo.

Não foram observadas evidências de associação com significância estatística entre os demais testes especiais (Palm Up, O’Brien e Yergason) e a lesão parcial de alto grau da CLB $(p>0,05)$.

Na - Tabela 5 foram avaliadas as associações entre a presença de dor à palpação do sulco intertubercular do úmero, os testes especiais do exame físico, os achados da ressonância magnética, e os achados intraoperatórios.

Nesse grupo específico de pacientes, em que todos apresentavam lesão do tendão do músculo supraespinal e algum grau de lesão do tendão da CLB, aqueles cuja lesão do tendão

Tabela 1 Medidas descritivas das variáveis numéricas na amostra de 50 pacientes

\begin{tabular}{|l|l|l|l|}
\hline Variável & Média (desvio padrão) & Mediana (1 ${ }^{\circ}$ quartil; $3^{\circ}$ quartil) & $\begin{array}{l}\text { Amplitude } \\
\text { mínima-máxima }\end{array}$ \\
\hline Avaliação clínica & & & $51-83$ \\
\hline Idade (anos) & $65,1(6,2)$ & $65(60 ; 69)$ & $2-240$ \\
\hline Tempo de sintomatologia (meses) & $30,7(41,4)$ & $12(7 ; 36)$ & $80-180$ \\
\hline Exame físico & & $160(140 ; 180)$ & $110-180$ \\
\hline Flexão ativa ( $\left.{ }^{\circ}\right)$ & $152,2(31,7)$ & $180(170 ; 180)$ & \\
\hline Flexã passiva ( $\left.{ }^{\circ}\right)$ & $173,2(13,8)$ & & $0,9-5,0$ \\
\hline $\begin{array}{l}\text { Avaliação radiológica: } \\
\text { ressonância magnética }\end{array}$ & & $2,9(1,5 ; 3,7)$ & \\
\hline $\begin{array}{l}\text { Tamanho da lesão do tendão do } \\
\text { músculo supraespinal (cm) }\end{array}$ & $2,8(1,2)$ & & \\
\hline
\end{tabular}


Tabela 2 Análises descritivas das variáveis categóricas da avaliação clínica e do exame físico

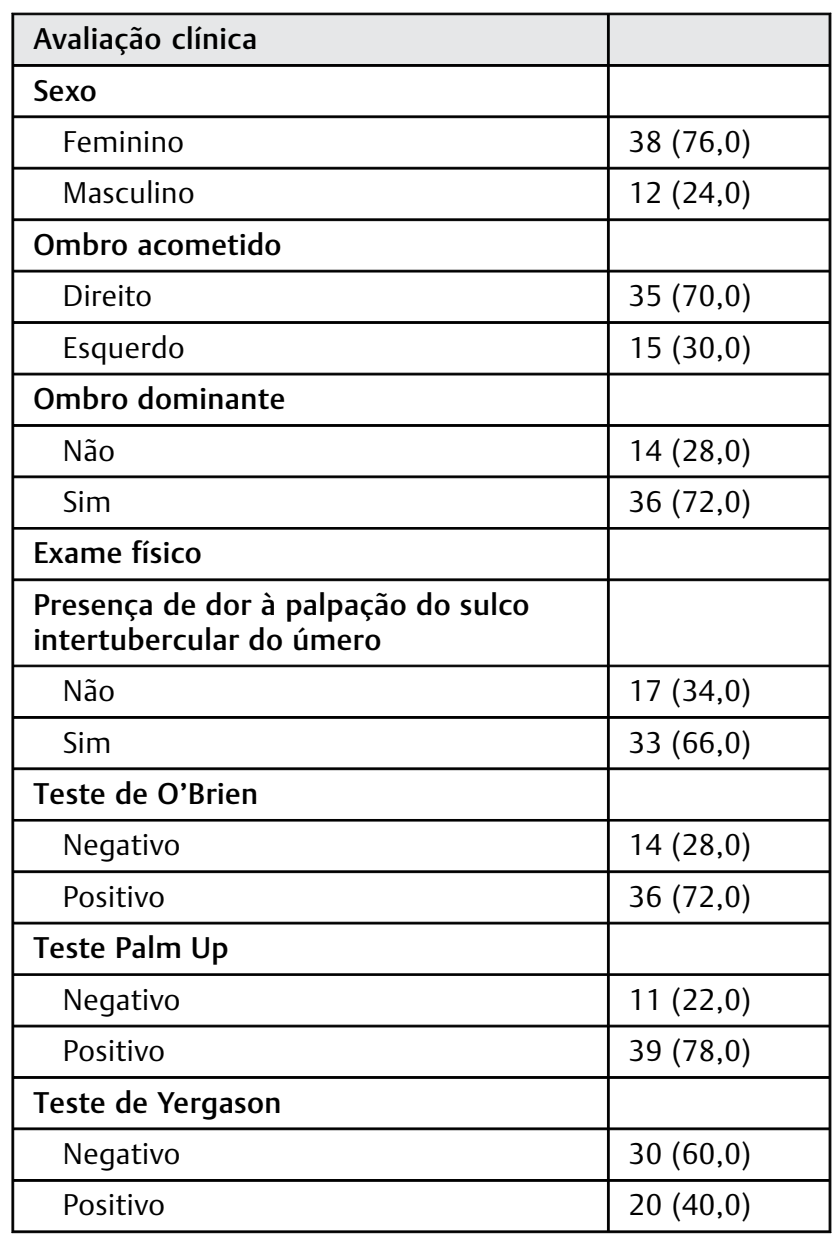

da CLB era de alto grau tiveram uma relação direta com o tamanho da lesão do supraespinal $(p<0,05)$, considerando que, para cada centímetro de lesão do tendão do músculo supraespinal, o paciente apresenta um risco 1,7 maior de ter uma lesão de alto grau da CLB. Desse modo, um paciente com lesão do tendão do músculo supraespinal de $1 \mathrm{~cm}$ e $3 \mathrm{~cm}$ de extensão tem, respectivamente, uma probabilidade de $41,4 \%$ e $67,1 \%$ de apresentar uma lesão significativa da CLB.

Na - Tabela 6 foram apresentadas as associações entre a presença de tendinopatia na CLB pela ressonância magnética e os testes do exame físico e os achados do intraoperatório; não foram observadas evidências de associação entre a tendinopatia presente na ressonância magnética e as variáveis analisadas $(p>0,05)$. Todavia, foi observada uma associação entre a lesão do tendão do músculo subescapular na ressonância magnética e a luxação da CLB encontrada na artroscopia $(p<0,001)$. Vale ressaltar que a probabilidade de um indivíduo com lesão total do tendão do músculo subescapular apresentar uma luxação da CLB no intraoperatório é de $91 \%$, ou um risco 55 vezes maior nos pacientes que apresentam essa lesão.

Aplicamos o teste de McNemar para avaliar a concordância entre as incidências encontradas nos testes do exame físico nas lesões avaliadas na ressonância magnética e observadas no
Tabela 3 Análises descritivas das variáveis categóricas da avaliação radiológica - ressonância magnética

\begin{tabular}{|c|c|}
\hline $\begin{array}{l}\text { Avaliação radiológica: } \\
\text { ressonância magnética }\end{array}$ & \\
\hline \multicolumn{2}{|l|}{ Lesão da cabeça longa do bíceps } \\
\hline Lesão total & $1(2,0)$ \\
\hline Negativo & $3(6,0)$ \\
\hline Positivo & $46(92,0)$ \\
\hline \multicolumn{2}{|c|}{ Lesão do tendão do músculo infraespinal } \\
\hline Não & $29(58,0)$ \\
\hline Sim & $21(42,0)$ \\
\hline \multicolumn{2}{|c|}{ Lesão do tendão do músculo subescapular } \\
\hline Não & $34(68,0)$ \\
\hline Sim & $16(32,0)$ \\
\hline \multicolumn{2}{|l|}{$\begin{array}{l}\text { Retração do tendão do } \\
\text { músculo supraespinal }\end{array}$} \\
\hline 1 & $8(16,0)$ \\
\hline II & $29(58,0)$ \\
\hline III & $9(18,0)$ \\
\hline Não & $4(8,0)$ \\
\hline \multicolumn{2}{|l|}{ Luxação da cabeça longa do bíceps } \\
\hline Não & $43(86,0)$ \\
\hline Sim & $7(14,0)$ \\
\hline \multicolumn{2}{|l|}{$\begin{array}{l}\text { Lesão superior labral tear from } \\
\text { anterior to posterior (SLAP) tipo II }\end{array}$} \\
\hline Não & $48(96,0)$ \\
\hline Sim & $2(4,0)$ \\
\hline
\end{tabular}

intraoperatório durante a artroscopia. Nessa perspectiva, pudemos observar que, na população estudada, a palpação do sulco intertubercular do úmero é um bom teste para detectar a lesão de alto grau do tendão da CLB. Da mesma forma, a ressonância magnética também apresentou concordância com a artroscopia para as lesões SLAP e para a luxação da CLB.

\section{Discussão}

Pacientes acometidos por doenças do tendão da CLB frequentemente relatam dor na região anterior do ombro, localizada no sulco intertubercular do úmero. Os sintomas podem ser difíceis de serem distinguidos dos das demais afecções do ombro, especialmente no caso das lesões do manguito rotador. ${ }^{16}$

Apesar da não significância estatística, encontramos uma positividade semelhante entre o teste de Palm Up e a presença de lesão de alto grau do tendão da CLB no intraoperatório: 78\% e 60\%, respectivamente. O teste Palm Up, de acordo com Bennett et al, ${ }^{17}$ é um teste com alta sensibilidade (90\%) para lesões macroscópicas do tendão da CLB, o que, em menor proporção, também foi observado em nosso estudo.

Observamos uma positividade de $72 \%$ no teste de O'Brien. Na ressonância magnética, observamos apenas $4 \%$ dos exames positivos para lesão SLAP. Já durante a avaliação 
334 Lesão do tendão do músculo supraespinal Carvalho et al.

Tabela 4 Análises descritivas das variáveis avaliadas no intraoperatório

\begin{tabular}{|c|c|}
\hline Proced & \\
\hline \multicolumn{2}{|c|}{ Hiperemia } \\
\hline Não & $6(12,0)$ \\
\hline Sim & $44(88,0)$ \\
\hline \multicolumn{2}{|c|}{ Achatamento } \\
\hline Não & $23(46,0)$ \\
\hline Sim & $27(54,0)$ \\
\hline \multicolumn{2}{|c|}{ Fibrilação } \\
\hline Não & $7(14,0)$ \\
\hline Sim & $43(86,0)$ \\
\hline \multicolumn{2}{|c|}{$\begin{array}{l}\text { Lesão parcial de alto grau da } \\
\text { cabeça longa do bíceps }\end{array}$} \\
\hline Não & $20(40,0)$ \\
\hline Sim & $30(60,0)$ \\
\hline \multicolumn{2}{|c|}{ Luxação da cabeça longa do bíceps } \\
\hline Não & $40(80,0)$ \\
\hline Sim & $10(20,0)$ \\
\hline \multicolumn{2}{|c|}{ Lesão do tendão do músculo subescapular } \\
\hline Não & $38(76,0)$ \\
\hline Sim & $12(24,0)$ \\
\hline \multicolumn{2}{|c|}{ Lesão da polia } \\
\hline Não & $39(78,0)$ \\
\hline Sim & $11(22,0)$ \\
\hline \multicolumn{2}{|c|}{$\begin{array}{l}\text { Lesão superior labral tear from } \\
\text { anterior to posterior (SLAP) tipo II }\end{array}$} \\
\hline Não & $47(94,0)$ \\
\hline Sim & $3(6,0)$ \\
\hline \multicolumn{2}{|c|}{ Classificação de Snyder para lesão SLAP } \\
\hline II & $3(6,0)$ \\
\hline Não & $47(94,0)$ \\
\hline
\end{tabular}

intraoperatória, encontramos 6\% dos pacientes com esta lesão. Utilizamos o teste de McNemar para avaliar a concordância entre o exame físico, a ressonância magnética, e a artroscopia. Como resultado, observamos uma discordância entre a positividade no teste de O'Brien tanto em relação à ressonância magnética quanto em relação à artroscopia, demonstrando que esse teste isolado não é adequado para o diagnóstico da lesão SLAP. ${ }^{18}$ Todavia, as incidências da lesão SLAP na ressonância magnética e na artroscopia apresentaram concordância estatisticamente significativa.

A literatura é controversa em relação à capacidade do teste de O'Brien, isoladamente, detectar a lesão SLAP. Ben Kibler et $\mathrm{al}^{12}$ demonstraram uma sensibilidade moderada (61\%) para o teste, em concordância com o estudo de Godinho et al, ${ }^{19}$ que observaram uma sensibilidade de $66,7 \%$. Esse teste não é capaz de reproduzir o movimento de peel back que ocorre na CLB para provocar o sintoma no paciente. Apesar

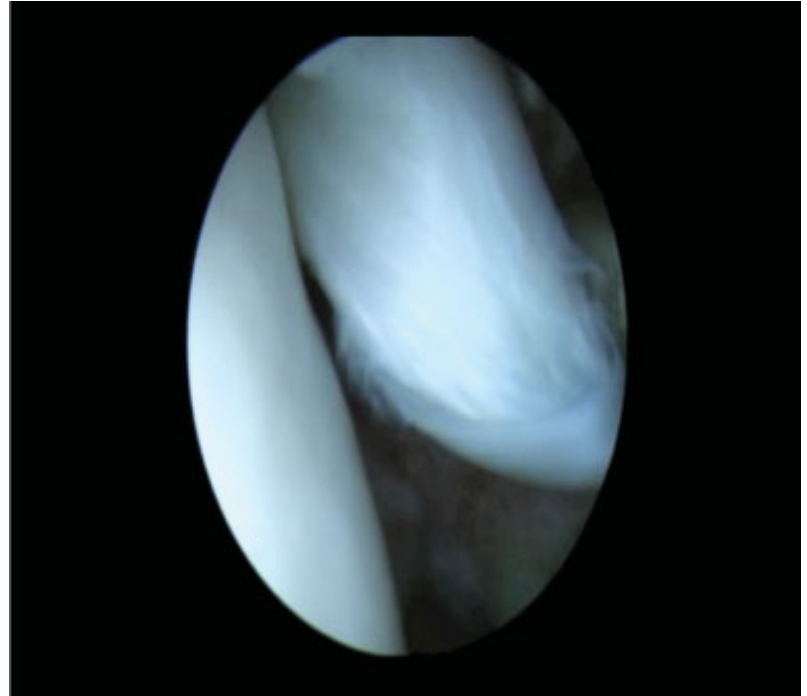

Fig. 3 Lesão de alto grau do tendão da cabeça longa do bíceps (CLB) acometendo mais de $50 \%$ da espessura do tendão.

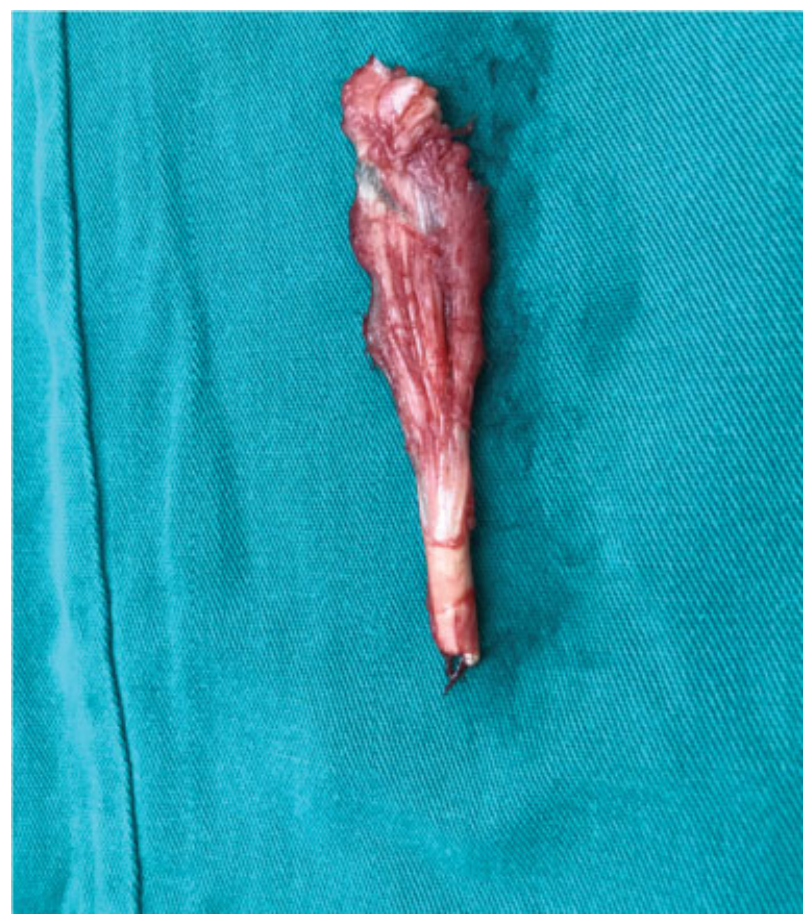

Fig. 4 Porção proximal do tendão da cabeça longa do bíceps (CLB) acometendo mais de $50 \%$ da espessura do tendão após tenotomia.

desse fato, o teste de O'Brien apresenta moderada capacidade de diagnosticar a lesão SLAP, ${ }^{16}$ o que não foi concordante com os nossos resultados. Por outro lado, outros autores concluíram que esse teste não é um indicador sensível para o diagnóstico, observando, ainda, uma grande incidência de pacientes falsos positivos, atribuindo a causa desse viés às lesões associadas no ombro (por exemplo, a lesão do manguito rotador). ${ }^{20,21}$

Nenhum teste isolado, aplicado para diagnosticar a lesão SLAP, é suficiente para o diagnóstico. Uma combinação entre os testes disponíveis pode aumentar a eficiência na 
Lesão do tendão do músculo supraespinal Carvalho et al. 335

Tabela 5 Associação entre dor e testes do exame físico, achados da ressonância magnética e achados intraoperatórios

\begin{tabular}{|c|c|c|c|c|}
\hline & & \multicolumn{2}{|c|}{$\begin{array}{l}\text { Dor à palpação do sulco } \\
\text { intertubercular do } \\
\text { úmero }\end{array}$} & \multirow[t]{2}{*}{ Valor de $f$} \\
\hline & & Não & Sim & \\
\hline \multicolumn{5}{|l|}{ Exame físico } \\
\hline \multirow[t]{2}{*}{ Teste de O’Brien } & Negativo $(n=14)$ & $6(42,9)$ & $8(57,1)$ & \multirow[t]{2}{*}{ ns } \\
\hline & Positivo $(n=36)$ & $11(30,6)$ & $25(69,4)$ & \\
\hline \multirow[t]{2}{*}{ Teste Palm Up } & Negativo $(n=11)$ & $6(54,5)$ & $5(45,5)$ & \multirow[t]{2}{*}{ ns } \\
\hline & Positivo $(n=39)$ & $11(28,2)$ & $28(71,8)$ & \\
\hline \multirow[t]{2}{*}{ Teste de Yergason } & Negativo $(n=30)$ & $12(40,0)$ & $18(60,0)$ & \multirow[t]{2}{*}{ ns } \\
\hline & Positivo $(n=20)$ & $5(25,0)$ & $15(75,0)$ & \\
\hline \multicolumn{5}{|l|}{ Ressonância magnética } \\
\hline \multirow[t]{2}{*}{ Tendinopatia da CLB } & Não $(n=3)$ & $1(33,3)$ & $2(66,7)$ & \multirow[t]{2}{*}{ ns } \\
\hline & $\operatorname{Sim}(n=46)$ & $16(34,8)$ & $30(65,2)$ & \\
\hline \multirow[t]{2}{*}{ Lesão do tendão do músculo infraespinal } & Não $(n=29)$ & $12(41,4)$ & $17(58,6)$ & \multirow[t]{2}{*}{ ns } \\
\hline & $\operatorname{Sim}(n=21)$ & $5(23,8)$ & $16(76,2)$ & \\
\hline \multirow[t]{2}{*}{ Lesão do tendão do músculo subescapular } & Não $(n=34)$ & $13(38,2)$ & $21(61,8)$ & \multirow[t]{2}{*}{ ns } \\
\hline & $\operatorname{Sim}(n=16)$ & $4(25,0)$ & $12(75,0)$ & \\
\hline \multirow[t]{2}{*}{ Luxação da CLB } & Não $(n=43)$ & $14(32,6)$ & $29(67,4)$ & \multirow[t]{2}{*}{ ns } \\
\hline & $\operatorname{Sim}(n=7)$ & $3(42,9)$ & $4(57,1)$ & \\
\hline \multirow[t]{2}{*}{ Lesão SLAP } & Não $(n=48)$ & $17(35,4)$ & $31(64,6)$ & \multirow[t]{2}{*}{ ns } \\
\hline & $\operatorname{Sim}(n=2)$ & $0(0,0)$ & $2(100,0)$ & \\
\hline \multicolumn{5}{|l|}{ Intraoperatório } \\
\hline \multirow[t]{2}{*}{ Hiperemia } & Não $(n=6)$ & $4(66,7)$ & $2(33,3)$ & \multirow[t]{2}{*}{ ns } \\
\hline & $\operatorname{Sim}(n=44)$ & $13(29,5)$ & $31(70,5)$ & \\
\hline \multirow[t]{2}{*}{ Achatamento } & Não $(n=23)$ & $7(30,4)$ & $16(69,6)$ & \multirow[t]{2}{*}{ ns } \\
\hline & $\operatorname{Sim}(n=27)$ & $10(37,0)$ & $17(63,0)$ & \\
\hline \multirow[t]{2}{*}{ Fibrilação } & Não $(n=7)$ & $3(42,9)$ & $4(57,1)$ & \multirow[t]{2}{*}{ ns } \\
\hline & $\operatorname{Sim}(n=43)$ & $14(32,6)$ & $29(67,4)$ & \\
\hline \multirow[t]{2}{*}{ Lesão parcial de alto grau da CLB } & Não $(n=20)$ & $12(60,0)$ & $8(40,0)$ & 0,003 \\
\hline & $\operatorname{Sim}(n=30)$ & $5(16,7)$ & $25(83,3)$ & \\
\hline Luxação da CLB & Não $(n=40)$ & $13(32,5)$ & $27(67,5)$ & ns \\
\hline & $\operatorname{Sim}(n=10)$ & $4(40,0)$ & $6(60,0)$ & \\
\hline Lesão do tendão do músculo subescapular & Não $(n=38)$ & $13(34,2)$ & $25(65,8)$ & ns \\
\hline & $\operatorname{Sim}(n=12)$ & $4(33,3)$ & $8(66,7)$ & \\
\hline Lesão da polia & Não $(n=39)$ & $13(33,3)$ & $26(66,7)$ & $\mathrm{ns}$ \\
\hline & $\operatorname{Sim}(n=11)$ & $4(36,4)$ & $7(63,6)$ & \\
\hline Lesão SLAP & Não $(n=47)$ & $16(34,0)$ & $31(66,0)$ & ns \\
\hline & $\operatorname{Sim}(n=3)$ & $1(33,3)$ & $2(66,7)$ & \\
\hline
\end{tabular}

Abreviaturas: CLB, cabeça longa do bíceps; SLAP, superior labral tear from anterior to posterior; ns.

Nota: Resultados expressos em frequências absoluta e relativa.

identificação da lesão, ainda que o resultado dessa associação seja pouco expressivo em relação a qualquer teste aplicado de maneira isolada. ${ }^{18}$

No total, $40 \%$ dos pacientes tiveram resultado positivo no teste de Yergason. Entretanto, somente em 20\% dos pacientes foi observada luxação do tendão da CLB à artroscopia, e 14\% foram diagnosticados na ressonância magnética. Da mesma maneira, quando aplicamos o teste de McNemar, observamos uma discordância entre a incidência do teste de Yergason e as incidências da lesão tanto na ressonância quanto na artroscopia, demonstrando que esse teste isolado não é adequado para o diagnóstico de luxação da CLB. Todavia, as incidências 
Tabela 6 Correlação da lesão da CLB na ressonância magnética com os testes do exame físico e achados intraoperatórios

\begin{tabular}{|c|c|c|c|c|}
\hline & & \multicolumn{2}{|c|}{ Lesão da CLB na RM } & \multirow[t]{2}{*}{ Valor de $p$} \\
\hline & & Não $(n=3)$ & $\operatorname{Sim}(n=47)$ & \\
\hline \multicolumn{5}{|l|}{ Exame físico } \\
\hline \multirow{2}{*}{$\begin{array}{l}\text { Presença de dor à palpação do sulco } \\
\text { intertubercular do úmero }\end{array}$} & Não & $1(33,3)$ & $16(34,0)$ & \multirow[t]{2}{*}{ ns } \\
\hline & Sim & $2(66,7)$ & $31(66,0)$ & \\
\hline \multirow[t]{2}{*}{ Teste de O'Brien } & Negativo & $2(66,7)$ & $12(25,5)$ & \multirow[t]{2}{*}{ ns } \\
\hline & Positivo & $1(33,3)$ & $35(74,5)$ & \\
\hline \multirow[t]{2}{*}{ Teste Palm Up } & Negativo & $0(0,0)$ & $11(23,4)$ & \multirow[t]{2}{*}{ ns } \\
\hline & Positivo & $3(100,0)$ & $36(76,6)$ & \\
\hline \multirow{2}{*}{ Teste de Yergason } & Negativo & $2(66,7)$ & $28(59,6)$ & \multirow[t]{2}{*}{ ns } \\
\hline & Positivo & $1(33,3)$ & $19(40,4)$ & \\
\hline \multicolumn{5}{|l|}{ Intraoperatório } \\
\hline \multirow[t]{2}{*}{ Hiperemia } & Não & $1(33,3)$ & $5(10,6)$ & \multirow[t]{2}{*}{ ns } \\
\hline & Sim & $2(66,7)$ & $42(89,4)$ & \\
\hline \multirow[t]{2}{*}{ Achatamento } & Não & $1(33,3)$ & $22(46,8)$ & \multirow[t]{2}{*}{ ns } \\
\hline & Sim & $2(66,7)$ & $25(53,2)$ & \\
\hline \multirow[t]{2}{*}{ Fibrilação } & Não & $0(0,0)$ & $7(14,9)$ & \multirow[t]{2}{*}{ ns } \\
\hline & Sim & $3(100,0)$ & $40(85,1)$ & \\
\hline \multirow[t]{2}{*}{ Lesão parcial de alto grau da CLB } & Não & $1(33,3)$ & $19(40,4)$ & \multirow[t]{2}{*}{ ns } \\
\hline & Sim & $2(66,7)$ & $28(59,6)$ & \\
\hline \multirow[t]{2}{*}{ Luxação da CLB } & Não & $2(66,7)$ & $38(80,9)$ & \multirow[t]{2}{*}{ ns } \\
\hline & Sim & $1(33,3)$ & $9(19,1)$ & \\
\hline \multirow[t]{2}{*}{ Lesão do tendão do músculo subescapular } & Não & $1(33,3)$ & $37(78,7)$ & \multirow[t]{2}{*}{ ns } \\
\hline & $\operatorname{Sim}$ & $2(66,7)$ & $10(21,3)$ & \\
\hline \multirow[t]{2}{*}{ Lesão da polia } & Não & $1(33,3)$ & $38(80,9)$ & \multirow[t]{2}{*}{ ns } \\
\hline & Sim & $2(66,7)$ & $9(19,1)$ & \\
\hline \multirow[t]{2}{*}{ Lesão SLAP } & Não & $3(100,0)$ & $44(93,6)$ & \multirow[t]{2}{*}{ ns } \\
\hline & Sim & $0(0,0)$ & $3(6,4)$ & \\
\hline \multirow[t]{2}{*}{ Classificação de Snyder para lesão SLAP } & II & $0(0,0)$ & $3(6,4)$ & \multirow[t]{2}{*}{ ns } \\
\hline & Não & $3(100,0)$ & $44(93,6)$ & \\
\hline
\end{tabular}

Abreviaturas: CLB, cabeça longa do bíceps; RM, ressonância magnética; SLAP, superior labral tear from anterior to posterior; ns.

da lesão na ressonância e na artroscopia também foram concordantes com a significância estatística.

Em consonância com nossos resultados em relação ao teste de Yergason, Ben Kibler et al $^{12}$ demonstraram em seu estudo que esse teste apresenta alta especificidade e baixa sensibilidade, sendo mais preciso para descartar a lesão do que para detectá-la.

Taylor et $a^{22}$ observaram que a palpação do sulco intertubercular do úmero e o teste O'Brien têm alta sensibilidade (97,8\% e 95,7\%, respectivamente). Por outro lado, os testes Palm Up e de Yergason são bastante específicos (86,7\% e $97,9 \%$, respectivamente), apesar de apresentarem baixa sensibilidade. Portanto, quando o teste de O’Brien e a palpação do sulco intertubercular do úmero forem negativos, podemos excluir com segurança uma lesão extra-articular da CLB.
Ao analisarmos nossos resultados em relação aos testes especiais descritos anteriormente, bem como os encontrados na literatura, observamos que os testes aplicados em conjunto têm maior êxito no diagnóstico do que quando aplicados isoladamente. $^{18}$

As lesãos do tendão da CLB são complexas e multifatoriais, sendo didaticamente definidas como lesões no complexo bíceps-labial, que são divididas em: lesões da inserção da CLB no labrum (lesões SLAP); lesões intra-articulares do corpo e da polia do tendão ; e lesões extra-articulares no sulco intertubercular do úmero. Tendo em vista essa interação entre as possiveis regiões que poderiam abrigar lesões dolorosas do tendão da CLB, foi proposto um conjunto de três testes para aumentar a precisão diagnóstica, em vez de usar os testes isolados para o diagnóstico. Propô-se aplicar os seguintes testes associados: palpação do sulco intertubercular do úmero; 
o teste do arremesso (com o braço abduzido a $90^{\circ}$, cotovelo fletido a $90^{\circ}$, e rotação externa máxima, o paciente inicia o movimento de arremesso contra resistência do examinador); e o teste de O'Brien. ${ }^{18,22}$

A ressonância magnética é o exame mais utilizado para diagnosticar lesões intra-articulares do tendão da CLB. Em nosso estudo, o exame foi positivo em $92 \%$ dos pacientes, sendo que $59,6 \%$ deles apresentavam lesão de alto grau da CLB (lesão acometendo mais de $50 \%$ do tendão). Observamos uma concordância de $90 \%$ entre a ressonância magnética e a artroscopia, considerando como sinais macroscópicos de lesão a hiperemia, o achatamento e a lesão parcial de alto grau.

Em contrapartida, Malavolta et $\mathrm{al}^{23}$ apresentaram em seu estudo uma sensibilidade moderada (67\%) e uma alta especificidade (98\%) para o exame de ressonância magnética. No referido estudo, foram avaliadas somente as rupturas totais do tendão da CLB, ao passo que em nosso estudo consideramos também sinais inflamatórios e as lesões parciais, o que poderia explicar a maior incidência de exames positivos.

Mohtadi et al $^{13}$ observaram uma menor prevalência de diagnóstico na ressonância magnética e uma menor concordância entre a ressonância magnética e a artroscopia, $66 \%$ e 37,7\%, respectivamente. Atribuímos essa discordância ao fato de a população avaliada em nosso estudo ser mais idosa (com uma média de idade de 65,1 anos, contrastando com uma média de idade de 46,2 anos do referido estudo), considerando que a incidência das lesões do tendão da CLB associadas às lesões do manguito rotador aumentam com a idade.

A única correlação com significância estatística entre o exame físico e a avaliação artroscópica encontrada em nosso estudo foi a dor à palpação do sulco intertubercular do úmero, observada em $83 \%$ dos pacientes cuja lesão acometia mais de $50 \%$ da espessura do tendão da CLB. A lesão parcial é a indicação mais comum para os procedimentos cirúrgicos: tenotomia e tenodese. ${ }^{24}$ Nessa perspectiva, o cirurgião já deve estar preparado para a possibilidade de intervenção no tendão da CLB caso a suspeita no exame físico seja confirmada no intraoperatório.

Atualmente, estudos histológicos têm questionado se realmente as alterações apresentadas nos exames de imagens, bem como as alterações morfológicas visualizadas na artroscopia, podem, de fato, caracterizar um processo inflamatório da CLB. Streit et $\mathrm{al}^{25}$ concluíram, em seu estudo, que a dor anterior do ombro não parece estar relacionada ao processo inflamatório da porção extra-articular do tendão da CLB na maioria dos casos. Dos 26 pacientes avaliados, somente 2 apresentavam alterações histológicas de inflamação crônica, e nenhum deles apresentou alteração histológica característica de processo inflamatório agudo.

A relação entre as lesões do manguito rotador e as lesões do tendão da CLB são bem conhecidas na literatura médica, ${ }^{15}$ e, apesar de vários estudos confirmarem sua relação anatômica, apenas alguns deles se dedicaram a uma investigação mais detalhada. ${ }^{26} \mathrm{~A}$ associação entre essas duas lesões varia na literatura entre $78,5 \%$ em um grupo amostral menor $(n=28),{ }^{24}$ e $22 \%$ em uma amostragem maior $(n=207)$, conforme observado por Braum et al. ${ }^{27}$

Lafosse et $\mathrm{al}^{21}$ observaram uma forte relação entre as lesões da CLB e o tamanho da lesão do manguito rotador. Desse modo, nosso estudo corrobora a literatura e acrescenta uma relação de risco ainda não descrita entre essas duas variáveis. Observamos que, para cada centímetro de lesão do tendão do músculo supraespinal, o paciente tem um risco 1,7 vezes maior de desenvolver uma lesão de alto grau do tendão da CLB. A incidência varia de $41,4 \%$ a $67,1 \%$ para as lesões de $1 \mathrm{~cm}$ e $3 \mathrm{~cm}$, respectivamente.

Pacientes submetidos à intervenção cirúrgica no bíceps concomitante ao reparo da lesão do manguito rotador obtiveram melhores resultados em relação ao reparo do manguito isolado. ${ }^{28}$ Chechia et $\mathrm{al}^{29}$ demonstraram, em seu estudo, uma satisfação de 93,4\% em pacientes submetidos ao reparo do manguito rotador associado à tenodese do tendão da CLB. Ikemoto et $\mathrm{al}^{30}$ observaram melhores resultados no grupo de pacientes submetidos à tenotomia associada à tenodese em relação à tenotomia isolada, ressaltando que os pacientes submetidos à tenotomia isolada também apresentaram resultados satisfatórios.

O estudo realizado apresentou limitações em relação à sua amostra, apesar da população de 50 pacientes ser compatível com a literatura nacional. Para um poder de teste de $50 \%$ e $90 \%$, necessitaríamos de uma amostra de 208 e 300 pacientes, respectivamente. Da mesma maneira, apontamos como fatores limitantes do nosso estudo a ausência de um grupo de controle com pacientes sem a lesão do tendão da CLB, bem como de uma população específica, composta, em sua maioria, por pacientes do sexo feminino com uma média de idade de 65,1 anos.

O fato de não termos encontrado associações significativas entre as variáveis não significa que elas não existam, o tamanho amostral pode ter sido o responsável pela não significância nas análises.

Conflito de Interesses

Os autores declaram não haver conflito de interesses

\section{Referências}

1 Rockwood CA Jr, Matsen 3rd, Frederick A. The shoulder. 4th ed. Philadelphia: Saunders Elsevier; 2009

2 Kessel L, Watson M. The painful arc syndrome. Clinical classification as a guide to management. J Bone Joint Surg Br 1977;59 (02):166-172

3 Itoi E, Kuechle DK, Newman SR, Morrey BF, An KN. Stabilising function of the biceps in stable and unstable shoulders. J Bone Joint Surg Br 1993;75(04):546-550

4 Warner JJ, McMahon PJ. The role of the long head of the biceps brachii in superior stability of the glenohumeral joint. J Bone Joint Surg Am 1995;77(03):366-372

5 Pagnani MJ, Deng XH, Warren RF, Torzilli PA, O'Brien SJ. Role of the long head of the biceps brachii in glenohumeral stability: a biomechanical study in cadavera. J Shoulder Elbow Surg 1996;5 (04):255-262

6 Codman EA. The Shoulder. Boston: Thomas Todd; 1934

7 DePalma AF. Surgery of the shoulder. Philadelphia: JB Lippincott; 1950 
8 Godinho GG, Mesquita FA, França FdeO, Freitas JM. “ROCAMBOLELIKE" biceps tenodesis: technique and results. Rev Bras Ortop 2015;46(06):691-696

9 Enad JG. Bifurcate origin of the long head of the biceps tendon. Arthroscopy 2004;20(10):1081-1083

10 Logal RJ. Rupture of the long tendon of the biceps brachii muscle. Clin Orthop Relat Res 1976;(121):217-221

11 Raney EB, Thankam FG, Dilisio MF, Agrawal DK. Pain and the pathogenesis of biceps tendinopathy. Am J Transl Res 2017;9(06): 2668-2683

12 Ben Kibler W, Sciascia AD, Hester P, Dome D, Jacobs C. Clinical utility of traditional and new tests in the diagnosis of biceps tendon injuries and superior labrum anterior and posterior lesions in the shoulder. Am J Sports Med 2009;37(09):1840-1847

13 Mohtadi NG, Vellet AD, Clark ML, et al. A prospective, doubleblind comparison of magnetic resonance imaging and arthroscopy in the evaluation of patients presenting with shoulder pain. J Shoulder Elbow Surg 2004;13(03):258-265

14 Anbar A, Emad Y, Zeinhom F, Ragab Y. Shoulder arthroscopy remains superior to direct MR arthrography for diagnosis of subtle rotator interval lesions. Eur J Orthop Surg Traumatol 2015;25(04):689-697

15 Neviaser TJ, Neviaser RJ, Neviaser JS, Neviaser JS. The four-in-one arthroplasty for the painful arc syndrome. Clin Orthop Relat Res 1982;(163):107-112

16 Ejnisman B, Monteiro GC, Andreoli CV, de Castro Pochini A. Disorder of the long head of the biceps tendon. $\mathrm{Br} \mathrm{J}$ Sports Med 2010;44(05):347-354

17 Bennett WF. Specificity of the Speed's test: arthroscopic technique for evaluating the biceps tendon at the level of the bicipital groove. Arthroscopy 1998;14(08):789-796

18 Taylor SA, Newman AM, Dawson C, et al. The “3-Pack" Examination is critical for comprehensive evaluation of the Biceps Labrum Complex and the Bicipital Tunnel: a prospective study. Arthroscopy 2017;33(01):28-38

19 Godinho GG, Freitas JM, França FO, Lopes AA, Milazzo AJ, Dal Zotto C. Efficiency of the evaluation, clinical (O'Brien test) and imaging (Arthro-MRI) methods in the diagnosis of shoulder SLAP lesions. Rev Bras Ortop 2006;41(11/12):461-466
20 Stetson WB, Templin K. The crank test, the O'Brien test, and routine magnetic resonance imaging scans in the diagnosis of labral tears. Am J Sports Med 2002;30(06):806-809

21 Lafosse L, Reiland Y, Baier GP, Toussaint B, Jost B. Anterior and posterior instability of the long head of the biceps tendon in rotator cuff tears: a new classification based on arthroscopic observations. Arthroscopy 2007;23(01):73-80

22 Taylor SA, Khair MM, Gulotta LV, et al. Diagnostic glenohumeral arthroscopy fails to fully evaluate the biceps-labral complex. Arthroscopy 2015;31(02):215-224

23 Malavolta EA, Assunção JH, Guglielmetti CL, de Souza FF, Gracitelli ME, Ferreira Neto AA. Accuracy of preoperative MRI in the diagnosis of disorders of the long head of the biceps tendon. Eur J Radiol 2015;84(11):2250-2254

24 Creech MJ, Yeung M, Denkers M, Simunovic N, Athwal GS, Ayeni OR. Surgical indications for long head biceps tenodesis: a systematic review. Knee Surg Sports Traumatol Arthrosc 2016;24(07): 2156-2166

25 Streit JJ, Shishani Y, Rodgers M, Gobezie R. Tendinopathy of the long head of the biceps tendon: histopathologic analysis of the extra-articular biceps tendon and tenosynovium. Open Access J Sports Med 2015;6:63-70

26 Redondo-Alonso L, Chamorro-Moriana G, Jiménez-Rejano JJ, López-Tarrida P, Ridao-Fernández C. Relationship between chronic pathologies of the supraspinatus tendon and the long head of the biceps tendon: systematic review. BMC Musculoskelet Disord 2014;15:377

27 Braun S, Horan MP, Elser F, Millett PJ. Lesions of the biceps pulley. Am J Sports Med 2011;39(04):790-795

28 Watson ST, Robbins CB, Bedi A, Carpenter JE, Gagnier JJ, Miller BS. Comparison of outcomes 1 year after Rotator Cuff Repair with and without concomitant biceps surgery. Arthroscopy 2017;33(11): 1928-1936

29 Checchia SL, Doneux PS, Miyazaki AN, et al. Biceps tenodesis associated with arthroscopic repair of rotator cuff tears. J Shoulder Elbow Surg 2005;14(02):138-144

30 Ikemoto RY, Pileggi PE, Murachovsky J, et al. Tenotomy with or without tenodesis of the long head of the biceps for arthroscopic repair of the rotator cuff. Rev Bras Ortop 2015;47(06):736-740 